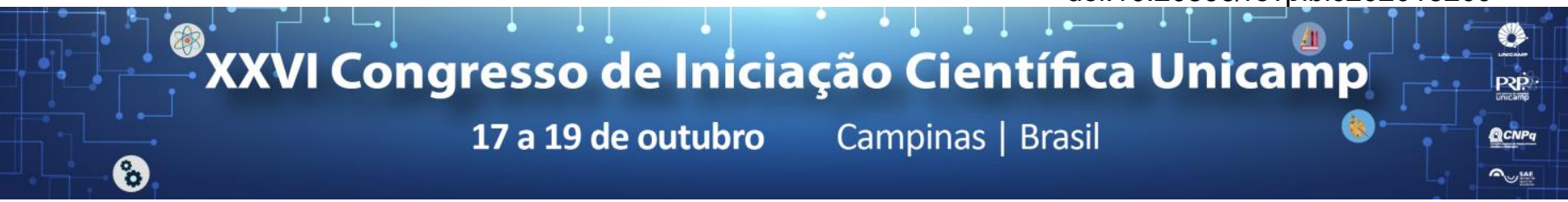

\title{
Miltefosine susceptibility of isolates of Leishmania (Leishmania) infantum from dogs of the municipality of Embu-Guaçu, Brazil
}

\author{
Bianca A. Ferreira*, Edite H. Y. Kanashiro, Mussya C. Rocha, Paulo C. Cotrim, Adriano C. Coelho
}

\begin{abstract}
Visceral leishmaniasis (VL) is a parasitic disease caused by the protozoan Leishmania (L.) infantum. In Brazil, the number of cases of the disease has increased in the last years. The treatment of leishmaniasis in Brazil consists of the use of pentavalent antimonials and amphotericin B. Recently, miltefosine has been shown to be highly effective against $\mathrm{VL}$ in Asia. Although, this drug is not used in the treatment of VL in Brazil, miltefosine is approved for use in the treatment of canine visceral leishmaniasis (CVL). In this study, we evaluate the susceptibility to miltefosine in vitro of isolates of L. (L.) infantum from dogs of municipality of Embu-Guaçu, located in the metropolitan region of the city of São Paulo.
\end{abstract}

Key words: visceral leishmaniasis, Leishmania infantum, miltefosine, drug susceptibility.

\section{Introduction}

Visceral leishmaniasis is a parasitic disease caused by the protozoan $L$. (L.) infantum. The disease is the most severe clinical form of leishmaniasis that can lead to death if it is not treated. In Brazil, about 3,000 new cases of the disease are reported annually, with an increasing number of cases in urban and periurban areas. Since VL is zoonotic in Brazil, domestic dogs constitute the main reservoir for the parasite, playing an essential role in transmission of disease to humans. The treatment of $\mathrm{VL}$ in Brazil consists in the use of pentavalent antimonials and amphotericin $B$, drugs that are considered expensive, toxic and that require parenteral administration. In CVL, the only drug used for treatment is miltefosine, a drug already used in the treatment of VL in Southeast Asia. In this study, we aim to evaluate the susceptibility to miltefosine in vitro of isolates of $L$. (L.) infantum from dogs of the municipality of Embu-Guaçu. Considering the potential of miltefosine be used in the chemotherapy of VL in the near future, it is urgent to investigate the susceptibility of $L$. (L.) infantum from dogs that are potential reservoirs of the disease in Brazilian endemic regions.

\section{Results and Discussion}

Previously, isolates were typed by Instituto Oswaldo Cruz, FIOCRUZ, RJ, Brazil according to the protocol described by Cupolillo et al., $1994^{1}$ and then confirmed by polymerase chain reaction (PCR) of $h s p 70$ gene followed by digestion with the restriction enzyme Hae III ${ }^{2}$ (Fig. $1 \mathrm{~A}$ and $1 \mathrm{~B}$ ). The in vitro susceptibility of isolates and the reference strains of $L$. (L.) infantum to miltefosine in promastigotes were determined as previously described ${ }^{3}$. The $\mathrm{EC}_{50}$ values of miltefosine against promastigotes ranged from $6,5 \pm 0,9$ to $34,14 \pm 1,56 \mu \mathrm{M}$ (Fig. $1 \mathrm{C})$. These findings suggest a moderate variation in miltefosine susceptibility of these isolates from dogs.

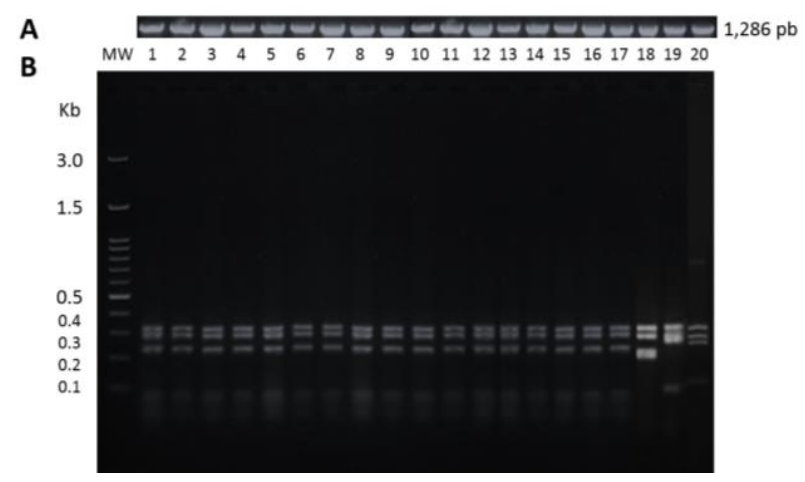

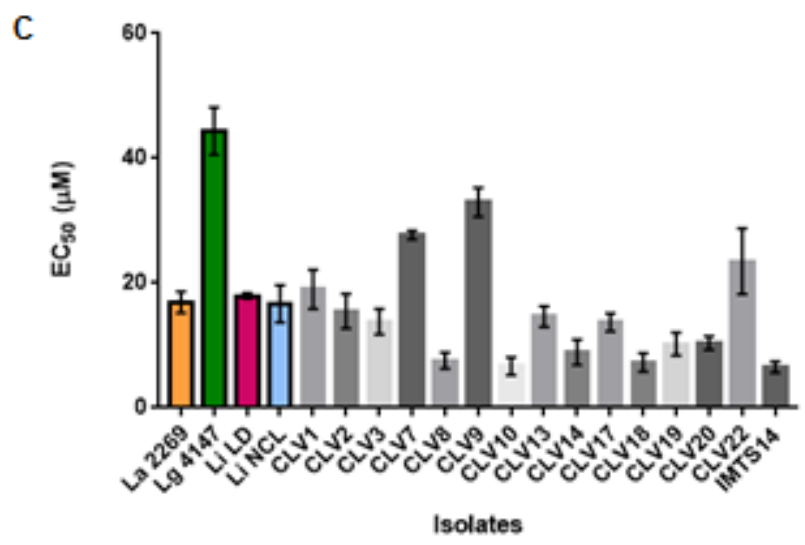

Figure 1. A. PCR amplification of the $h s p 70$ gene $(1,286 \mathrm{bp})$. B. Digestion of the PCR amplified product with the restriction enzyme Haelll. Legend: 1- L. (L.) infantum LD; 2- L. (L.) infantum NCL; 3CVL1; 4- CVL2; 5- CVL3; 6- CVL7; 7- CVL8; 8- CVL9; 9- CVL10; 10CVL13; 11- CVL14; 12- CVL17; 13- CVL18; 14- CVL19; 15- CVL20; 16CVL22;17- IMTS 14; 18- L. (L.) amazonensis (MHOM/BR/1973/M2269); 19- L. (V.) braziliensis (MHOM/BR/1975/M2903); 20- L. (V.) guyanensis (MHOM/BR/1975/M4147). C. EC E $_{50}$ values determined against promastigotes of isolates from dogs.

\section{Conclusions}

The results obtained in this study will contribute to evaluate the potential of miltefosine against isolates of $L$. (L.) infantum from domestic dogs, the most important reservoir of $V L$ in urban areas in Brazil.

\section{Acknowledgement}

We thank Silvia R. B. Uliana from Universidade de São Paulo for providing the $L$. (L.) infantum reference strains. This work is funded by Fundação de Amparo à Pesquisa do Estado de São Paulo (FAPESP 2016/21171-6). BAF is supported by FAPESP fellowship (2017/18488-0).

\footnotetext{
1 Cupolillo, E.; Grimaldi, G.Jr.; Momen, H. Am J. A general classification of New World Leishmania using numerical zymotaxonomy. Trop Med Hyg. 1994 50(3):296-311.

2 Montalvo, A.M.J.; Fraga, et al. Three new sensitive and specific heat-shock protein 70 PCRs for global Leishmania species identification. Eur J Clin Microbiol Infect Dis. 2012, 31(7): 1453-61.

3 Espada, C.R; Ribeiro, D. F.; Dorta, M.L; Pereira, L.I.A; Carvalho, E.M; Machado, P.R; Schriefer,A.;Yokoyama,Y.J.K.U; Coelho,A.C.;Uliana,S.R.B Susceptibility to Miltefosine in Brazilian Clinical Isolates of Leishmania (Viannia) braziliensis.. Am J Trop Med Hyg 2017 96(3):656-659
} 